\title{
MCCARD: MONTE CARLO CODE FOR ADVANCED REACTOR DESIGN AND ANALYSIS
}

\author{
HYUNG JIN SHIM ${ }^{1 *}$, BEOM SEOK HAN ${ }^{2}$, JONG SUNG JUNG ${ }^{3}$, HO JIN PARK ${ }^{4}$, and CHANG HYO KIM ${ }^{1}$ \\ ${ }^{1}$ Seoul National University \\ 1 Gwanak-ro, Gwanak-gu, Seoul 151-742, Korea \\ ${ }^{2}$ Korea Institute of Nuclear Safety \\ 62 Gwahak-ro, Yuseong-gu, Daejeon 305-338, Korea \\ ${ }^{3}$ KEPCO Nuclear Fuel \\ 989 Daedeok-daero, Yuseong-gu, Daejeon 305-353, Korea \\ ${ }^{4}$ Korea Atomic Energy Research Institute \\ 1045 Daedeok-daero, Yuseong-gu, Daejeon 305-353, Korea \\ "Corresponding author. E-mail : shimhj@snu.ac.kr
}

Invited March 23, 2011

Received December 14, 2011

Accepted for Publication January 08, 2012

McCARD is a Monte Carlo (MC) neutron-photon transport simulation code. It has been developed exclusively for the neutronics design of nuclear reactors and fuel systems. It is capable of performing the whole-core neutronics calculations, the reactor fuel burnup analysis, the few group diffusion theory constant generation, sensitivity and uncertainty (S/U) analysis, and uncertainty propagation analysis. It has some special features such as the anterior convergence diagnostics, real variance estimation, neutronics analysis with temperature feedback, $\mathrm{B}_{1}$ theory-augmented few group constants generation, kinetics parameter generation and MC S/U analysis based on the use of adjoint flux. This paper describes the theoretical basis of these features and validation calculations for both neutronics benchmark problems and commercial PWR reactors in operation.

KEYWORDS : McCARD, Monte Carlo Particle Transport, Few Group Constant, Sensitivity and Uncertainty, Gen-IV Reactors

\section{INTRODUCTION}

McCARD is a Monte Carlo (MC) neutron-photon transport simulation code designed exclusively for neutronics analyses of various nuclear reactor and fuel systems. Since its predecessor MCNAP [1] was first introduced in 1999 as a MC burnup analysis tool with an ORIGEN2-type [2] fuel depletion equation solver, it has evolved to a versatile MC tool that is capable of conducting whole-core neutronics calculations, few group diffusion theory constants (few group constants) generation, and uncertainty propagation calculations. In the course of its evolution, a wide range of nuclear systems such as SMART [3], PMR-200 [4], KALIMER-600 [5], fusion blankets [6], subcritical systems like HYPER [7], YALINA [8], and IPEN/MB-01 [9] have been subjected to the neutronics analyses. The R\&D efforts to meet both functional and non-functional requirements for these analyses have played crucial roles in developing McCARD into its current status.

McCARD estimates neutronics design parameters of a nuclear reactor or a fuel system such as effective multiplication factor $\left(k_{\text {eff }}\right)$, neutron flux and current, fission power, etc. by using continuous-energy cross section libraries and detailed geometrical data of the system. The neutron and photon cross section libraries are processed by NJOY [10]. A combination of hierarchical geometry options and the Python scripts [11] are adopted to input complex geometrical data efficiently and at the same time with as much simplicity as possible. The McCARD burnup analysis is performed with a built-in depletion equation solver based on the matrix exponential method [2, 12]. The predictor-corrector [13] calculation option is available for more accurate burnup analyses of nuclear reactor cores having strong absorbers like burnable poison rods (BPRs). McCARD can handle the double heterogeneity of the High Temperature Gas-cooled Reactor (HTGR) cores by direct sampling of the spatial distribution of fuel particles or pebbles from the random distribution function [14].

McCARD is written in an object-oriented programming language, $\mathrm{C}++$, and it is relatively easy to maintain, modify, and expand. It has incorporated many recent advances in the MC particle transport analysis methodology as special computational modules. Listed below are some 
of the special features implemented in McCARD.

- McCARD is equipped with anterior stopping criteria [15] which detect the onset of the fundamental mode fission source distribution (FSD), terminate the inactive cycle calculations, and automatically initiate active cycle eigenvalue calculations. The McCARD is also equipped with the posterior fission source convergence criterion based on the Shannon entropy [16] which makes it possible to diagnose whether the active cycle FSDs from the use of the anterior criteria are truly stationary, if users opt to do so.

- The sample variances of the tallied neutronics parameters in the MC eigenvalue calculations are biased because of intercycle correlations of the FSD. There are several studies on how to estimate the biases of the sample variances to determine the real variances of the tallied parameters. McCARD offers four different methods currently available to estimate the biases of the sample variances or the real variances; the Gelbard's batch method [17], the Ueki's method [18], the FSD's intercycle correlation method [19], and the history-based batch method [20].

In addition to the static neutronics design parameter calculation module, McCARD has the kinetics parameter calculation module designed to calculate the adjoint-weighted kinetics parameters such as the effective delayed neutron fraction, $\beta_{\text {eff }}$, and the prompt neutron generation time, $\Lambda$. The adjoint flux needed for this module is the self-consistent adjoint flux that can be determined during the $\mathrm{MC}$ forward calculations [21].

McCARD provides a few group constants generation module which aims at producing critical spectrum weighted few group constants for a nuclear fuel system such as a fuel pin cell or a fuel assembly as a function of fuel depletion using the $\mathrm{B}_{1}$ theory-augmented $\mathrm{MC}$ method [22]. One of the salient features of this module is the ability to model the random distribution of the fuel particles in the HTGR fuel compact or pebbles [14]. McCARD has an uncertainty quantification module aimed at quantifying both uncertainties of the tallied parameters from a single MC step neutronics calculations for a given nuclear system and the propagated uncertainties of the parameters from a combination of the $\mathrm{MC}$ and the deterministic method calculations like the $\mathrm{MC}$ depletion analysis and few group constants generation. The module makes the best of the adjointbased MC perturbation technique [23], and is applicable to the $\mathrm{S} / \mathrm{U}$ analysis aimed at assessing effects of the uncertainties of evaluated nuclear cross section data on the criticalities of criticality benchmark problems as well as neutronics design parameters of research or commercial power reactors, transmutation reactors or fuel systems [24]. It can also be applicable to estimating the uncertainties of the nuclide number densities of the burnt fuel assembly (FA) [25] and those of burnup- dependent few group constants as functions of uncertainties of uncertain nuclear cross section and nuclide number density input data to McCARD [26].

What follows is a brief account of the layout of this paper. Followed by the introduction, Section 2 gives a brief description of the general features of and some numerical results from McCARD. Section 3 describes the theoretical basis of special features of McCARD such as the FSD convergence diagnostics, the real variance estimations, and the temperature feedback calculations, which are essential for the whole-core MC calculations. Section 4 describes the $\mathrm{B}_{1}$ theory-augmented $\mathrm{MC}$ method used to develop the few group constants generation module of McCARD, and presents the burnup-dependent few group constants of pressurized water reactor (PWR) fuel assemblies. Section 5 presents both the mathematical basis of the uncertainty quantification module and some of the numerical results. The latter includes the $\mathrm{S} / \mathrm{U}$ analysis for effects of the evaluated nuclear data uncertainties on the criticality estimates of benchmark problems, the uncertainty propagation analysis of MC depletion analysis of a PWR FA, and the effects of nuclear cross section and nuclide number density uncertainties on the burnup-dependent few group constants of PWR.

\section{GENERAL FEATURES}

\subsection{Overview}

McCARD can be used for both source-mode and eigenvalue calculations through the MC particle simulation algorithms [27-29] for neutrons and neutron-induced photons. It utilizes continuous-energy neutron and photon cross section libraries and thermal scattering data of molecules or crystalline solids for thermal neutrons. In addition to the continuous energy $\mathrm{MC}$ calculations, the multi-group $\mathrm{MC}$ calculations can also be conducted by using multi-group neutron cross-section libraries. The energy-dependent fission product yield, and the decay chain data can be easily modified by editing external library files.

McCARD inputs are written in text mode with freeform syntax in which keywords and parameters need not be placed in specific columns and whitespace is just used as a delimiter. System geometry is inputted as a sum of three-dimensional unit cells, each of which is represented by the surface or boundary representation (B-rep) modeling. As a way to input geometrical data of a complex system efficiently, McCARD adopts a hierarchical modeling option that makes the best of hierarchical tiers called composition cells. For example, users can input geometrical data of a nuclear reactor core first by providing geometrical definitions of fuel pins and the layout of assemblies using the pre-defined fuel pins and then by describing the loading pattern of the pre-defined assemblies. A repeated geometry 
can be easily modeled by using the Python scripts [11]. The Python interpreter imbedded in McCARD enhances greatly the applicability of a generalized input file.

McCARD is capable of the burnup analysis by using the built-in depletion equation solver module. Unlike many existing MC burnup analysis codes, therefore, it is not necessary to couple the MC neutronics analysis modules with an external depletion code. As a result, a user can easily activate the burnup analysis module by declaring cells subject to depletion and specifying a thermal power and burnup steps. The code then automatically generates burnup tallies for the depletion cells. It provides three options for the predictor-corrector algorithm: no corrector, semi-predictor-corrector, and full predictor-corrector. In the semi-predictor-corrector option [13], the tally calculations for the corrected number densities are not conducted to save the computation time. In the full predictor-corrector option, however, they are conducted for an accurate analysis. A simplified flow diagram of the McCARD burnup calculations for the semi-predictor-corrector algorithm is shown in Fig. 1.

Tallies for various nuclear parameters can be calculated by track-length and collision estimators. The parallel computation capability of McCARD is implemented using the MPI [30] libraries. A load balancing algorithm by using a supervisor processor [31] highly augments parallel computational efficiency.

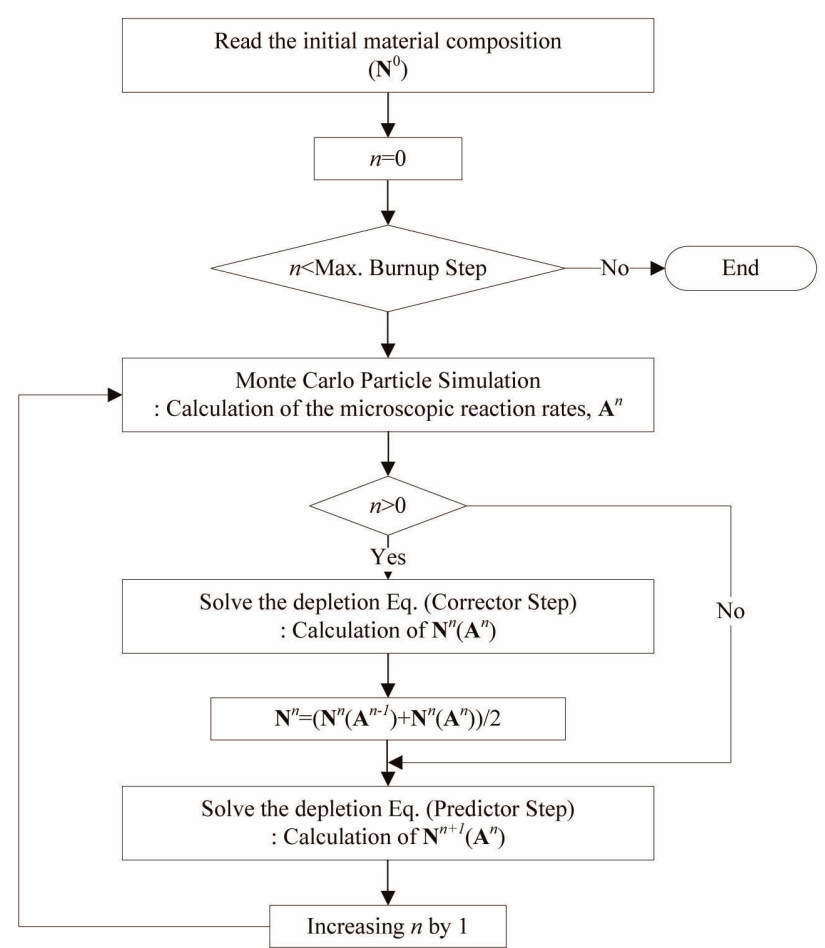

Fig. 1. Flowchart of the McCARD Burnup Calculations

\subsection{Validation}

The static and kinetics neutronics analysis capabilities of McCARD are tested against well-publicized neutronics benchmark problems such as International Criticality Safety Benchmark Experimental Problem (ICSBEP) [32], the Venus PWR engineering experiments [33-35], and the burnup benchmark problem suite for the reactor physics studies of next-generation fuels of light water reactors [36]. The measurement data available as well as computations of internationally recognized $\mathrm{MC}$ and deterministic codes are used to present the degree of computational accuracy of McCARD analyses.

Table 1 shows a comparison of experiments and McCARD estimates on the criticality of 28 criticality benchmark problems selected from ICSBEP which include 11 Plutonium (PU), 7 highly enriched Uranium (HEU), 1 intermediate enriched Uranium (IEU), 1 low enriched uranium (LEU), 7 Uranium-233 (U233), and 1 mixed Plutonium-Uranium (MIX) systems. The McCARD eigenvalue calculations are obtained on 1,050 cycles including 50 inactive cycles with 100,000 neutron histories per cycle. Table 1 lists the results only for the two cases of using ENDF/B-VII.0 and ENDF/B-VI.8 nuclear cross section libraries as the continuous energy cross section input data. The word, 'SD,' stands for the standard deviation. Considering the experimental uncertainties, $\Delta k_{\text {eff }}$, the McCARD results agree well with the measurements.

The VENUS experiments [33-35] are analyzed to examine the accuracy of the McCARD on pin power estimations. Figure 2 displays McCARD estimates on typical 48 pin powers of VENUS-1 core [33] in comparison with the measurements. The root mean square (RMS) and maximum relative errors of the McCARD analysis using ENDF/B-VII.0 cross section libraries for all the 324 pin powers are shown to be $1.71 \%$ and $7.83 \%$, respectively. The McCARD analyses for VIP-PWR [34] and the VENUS-2 [35] have shown 3.1\% and 2.8\% RMS errors, respectively, and they are available in Ref. 37.

The $\mathrm{UO}_{2}$ fuel pin cell and the $\mathrm{PWR} \mathrm{UO}_{2}$ FA problems in the burnup benchmark problem suite [36] for studying the physics of next-generation fuels of light water reactors are analyzed with ENDF/B-VI.8 and ENDF/B-VII.0 libraries as continuous cross section input data to McCARD. Figures 3 and 4 compare the McCARD estimates on burnup dependent $k_{\text {inf }}$ with those from other computations by HELIOS [13], CASMO [38], and MVP-BURN [39] codes for the $\mathrm{UO}_{2}$ fuel pin cell and FA problems, respectively. McCARD calculations for the two problems show very good agreements with results from the three other codes mentioned above, except for $k_{\text {inf }}$ of the FA problem from MVP-BURN. The maximum deviation of the SD's of two McCARD estimates on $k_{\text {inf }}$ of the pin cell problem with ENDF/B-VI.8 and ENDF/B-VII.0 libraries is about $21 \mathrm{pcm}$.

McCARD has the capability to estimate the point kinetics parameter such as the effective delayed neutron 
Table 1. McCARD Estimates on $k_{\text {eff }}$ 's for ICSBEP Benchmark Problems

\begin{tabular}{|c|c|c|c|}
\hline \multirow{2}{*}{ Handbook ID } & \multirow{2}{*}{$\begin{array}{c}\text { Benchmark } \\
k_{\text {eff }}(\text { Unc.) }\end{array}$} & \multicolumn{2}{|c|}{ McCARD $k_{\text {eff }}(\mathrm{SD})$} \\
\hline & & ENDF/B-VII & ENDF/B-VI \\
\hline PU-MET-FAST-001 & $1.0000(0.0020)$ & $0.99999(0.00006)$ & $0.99751(0.00006)$ \\
\hline PU-MET-FAST-002 & $1.0000(0.0020)$ & $1.00003(0.00006)$ & $0.99829(0.00006)$ \\
\hline PU-MET-FAST-019 & $0.9992(0.0015)$ & $0.99832(0.00006)$ & $1.00137(0.00006)$ \\
\hline PU-MET-FAST-020 & $0.9993(0.0017)$ & $0.99879(0.00006)$ & $0.99890(0.00006)$ \\
\hline PU-MET-FAST-021, case a & $1.0000(0.0026)$ & $1.00610(0.00007)$ & $1.00711(0.00007)$ \\
\hline PU-MET-FAST-021, case b & $1.0000(0.0026)$ & $0.99613(0.00006)$ & $0.99510(0.00006)$ \\
\hline PU-MET-FAST-022 & $1.0000(0.0021)$ & $0.99859(0.00006)$ & $0.99619(0.00006)$ \\
\hline PU-SOL-THERM-011, case 18-1 & $1.0000(0.0052)$ & $0.99411(0.00008)$ & $0.99527(0.00008)$ \\
\hline PU-SOL-THERM-011, case 18-6 & $1.0000(0.0052)$ & $1.00004(0.00008)$ & $1.00053(0.00008)$ \\
\hline PU-SOL-THERM-011, case 16-5 & $1.0000(0.0052)$ & $1.00630(0.00009)$ & $1.00626(0.00009)$ \\
\hline PU-SOL-THERM-011, case 16-1 & $1.0000(0.0052)$ & $1.01202(0.00009)$ & $1.01237(0.00009)$ \\
\hline HEU-MET-FAST-001 & $1.0000(0.0010)$ & $0.99992(0.00006)$ & $0.99649(0.00006)$ \\
\hline HEU-MET-FAST-028 & $1.0000(0.0030)$ & $1.00299(0.00007)$ & $1.00156(0.00007)$ \\
\hline HEU-SOL-THERM-013, case 1 & $1.0012(0.0026)$ & $0.99830(0.00006)$ & $0.99872(0.00005)$ \\
\hline HEU-SOL-THERM-013, case 2 & $1.0007(0.0036)$ & $0.99754(0.00006)$ & $0.99788(0.00006)$ \\
\hline HEU-SOL-THERM-013, case 3 & $1.0009(0.0036)$ & $0.99410(0.00006)$ & $0.99425(0.00006)$ \\
\hline HEU-SOL-THERM-013, case 4 & $1.0003(0.0036)$ & $0.99555(0.00007)$ & $0.99569(0.00006)$ \\
\hline HEU-SOL-THERM-032 & $1.0015(0.0026)$ & $0.99909(0.00004)$ & $0.99857(0.00004)$ \\
\hline IEU-MET-FAST-007 & $1.0045(0.0007)$ & $0.99929(0.00005)$ & $1.00908(0.00005)$ \\
\hline LEU-COMP-THERM-077 & $1.0003(0.0010)$ & $1.00363(0.00007)$ & $0.99899(0.00008)$ \\
\hline U233-MET-FAST-001 & $1.0000(0.0010)$ & $0.99953(0.00006)$ & $0.99284(0.00006)$ \\
\hline U233-MET-FAST-002 & $1.0000(0.0010)$ & $0.99988(0.00006)$ & $0.99614(0.00006)$ \\
\hline U233-MET-FAST-003 & $1.0000(0.0010)$ & $0.99988(0.00006)$ & $0.99633(0.00006)$ \\
\hline U233-MET-FAST-004 & $1.0000(0.0007)$ & $1.00513(0.00006)$ & $1.00273(0.00006)$ \\
\hline U233-MET-FAST-005 & $1.0000(0.0030)$ & $0.99427(0.00006)$ & $1.00466(0.00006)$ \\
\hline U233-MET-FAST-006 & $1.0000(0.0010)$ & $0.99959(0.00007)$ & $1.00006(0.00007)$ \\
\hline U233-SOL-THERM-008 & $1.0006(0.0029)$ & $1.00145(0.00004)$ & $0.99718(0.00004)$ \\
\hline MIX-MET-FAST-008, case 1 & $0.9920(0.0063)$ & $0.99230(0.00005)$ & $0.99374(0.00005)$ \\
\hline
\end{tabular}

fraction, $\beta_{\text {eff }}$, and the prompt neutron generation time, $\Lambda$, using the self-consistent adjoint flux which can be computed during the MC forward simulations, the validity of which is demonstrated [21] via the true values for infinite homogenous two-group problems and with measurements for several critical facility systems. Table 2 shows a comparison of McCARD estimates on $\beta_{e f f}$ and $\beta_{e f f} / \Lambda$ and their measurements for Godiva and TCA $[40,41]$. McCARD results show that it can predict $\beta_{\text {eff }}$ and $\beta_{\text {eff }} / \Lambda$ within a $3 \%$ error. 


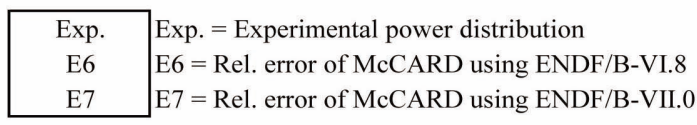

\begin{tabular}{||l|c|c|c|c||}
\hline 1.018 & 1.188 & 1.292 & 1.221 & \\
$3.53 \%$ & $3.42 \%$ & $-1.65 \%$ & $-1.41 \%$ & Pyrex \\
$3.50 \%$ & $2.99 \%$ & $-2.33 \%$ & $-1.81 \%$ & \\
\hline 0.970 & 1.162 & 1.258 & 1.219 & 1.202 \\
$1.76 \%$ & $2.78 \%$ & $0.01 \%$ & $1.55 \%$ & $-1.54 \%$ \\
$1.42 \%$ & $2.16 \%$ & $-0.53 \%$ & $1.38 \%$ & $-1.65 \%$ \\
\hline 0.963 & 1.162 & 1.274 & 1.226 & 1.206 \\
$0.61 \%$ & $0.69 \%$ & $-2.35 \%$ & $0.22 \%$ & $-2.28 \%$ \\
$0.09 \%$ & $0.26 \%$ & $-2.79 \%$ & $-0.07 \%$ & $-2.40 \%$ \\
\hline 0.958 & 1.149 & 1.245 & 1.168 & \\
$1.30 \%$ & $1.72 \%$ & $-1.57 \%$ & $0.33 \%$ & Pyrex \\
$0.66 \%$ & $0.77 \%$ & $-2.18 \%$ & $0.13 \%$ & \\
\hline 0.955 & 1.149 & 1.241 & 1.200 & 1.191 \\
$2.71 \%$ & $1.17 \%$ & $-0.69 \%$ & $1.75 \%$ & $-1.16 \%$ \\
$1.39 \%$ & $0.52 \%$ & $-1.87 \%$ & $1.28 \%$ & $-1.43 \%$ \\
\hline \hline
\end{tabular}

3.3 w/o enrichment region

\begin{tabular}{||l|l|l|l|l||}
\hline 0.417 & 0.366 & 0.320 & 0.277 & 0.228 \\
$1.02 \%$ & $3.85 \%$ & $4.49 \%$ & $4.91 \%$ & $4.92 \%$ \\
$1.32 \%$ & $3.48 \%$ & $4.46 \%$ & $4.49 \%$ & $5.36 \%$ \\
\hline 0.496 & 0.457 & 0.392 & 0.341 & 0.274 \\
$2.88 \%$ & $0.48 \%$ & $3.85 \%$ & $3.02 \%$ & $2.83 \%$ \\
$2.72 \%$ & $0.73 \%$ & $3.64 \%$ & $3.39 \%$ & $3.17 \%$ \\
\hline 0.572 & 0.509 & 0.461 & 0.373 & 0.312 \\
$0.00 \%$ & $1.63 \%$ & $-0.25 \%$ & $6.51 \%$ & $1.84 \%$ \\
$0.19 \%$ & $1.36 \%$ & $-0.30 \%$ & $6.47 \%$ & $2.25 \%$ \\
\hline 0.623 & 0.574 & 0.498 & 0.426 & 0.342 \\
$0.75 \%$ & $-1.18 \%$ & $1.09 \%$ & $1.89 \%$ & $2.05 \%$ \\
$0.61 \%$ & $-1.31 \%$ & $1.27 \%$ & $2.74 \%$ & $1.57 \%$ \\
\hline 0.680 & 0.613 & 0.550 & 0.463 & 0.365 \\
$-0.14 \%$ & $0.59 \%$ & $-0.69 \%$ & $1.49 \%$ & $2.91 \%$ \\
$-0.02 \%$ & $0.06 \%$ & $-1.12 \%$ & $1.56 \%$ & $2.91 \%$ \\
\hline \hline
\end{tabular}

$4.0 \mathrm{w} / \mathrm{o}$ enrichment region

Fig. 2. Comparison of Pin Power Distributions for VENUS-1 Critical Facility

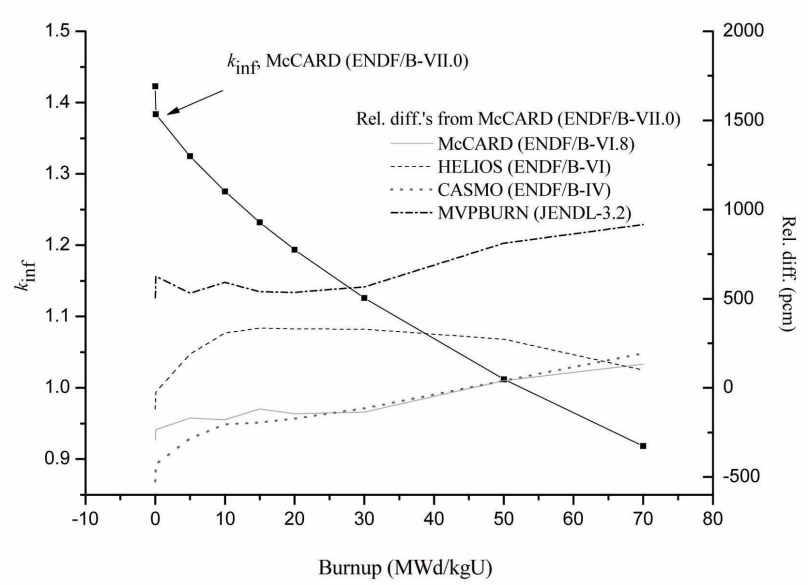

Fig. 3. Comparison of $\mathrm{k}_{\mathrm{inf}}$ 's for the $\mathrm{UO}_{2}$ Fuel Pin Problem in the Burnup Benchmark Suite

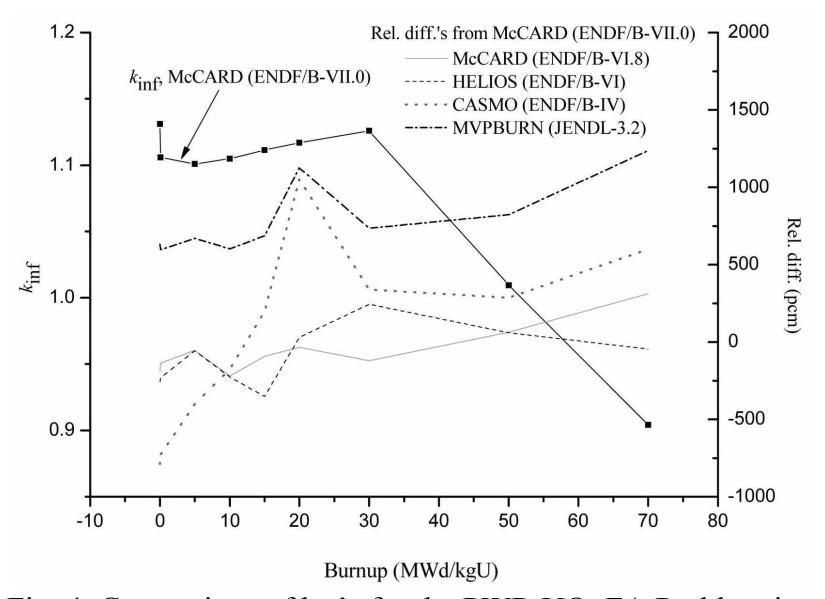

Fig. 4. Comparison of $\mathrm{k}_{\text {inf }}$ 's for the $\mathrm{PWR} \mathrm{UO}_{2}$ FA Problem in the Burnup Benchmark Suite

Table 2. Comparison of McCARD Estimates on $\beta_{\text {eff's }}$ and $\beta_{\text {eff }} / \Lambda$ 's with Measurements for Godiva and TCA

\begin{tabular}{|c|c|c|c|c|c|c|c|}
\hline Facility & Core Name & $\left(\beta_{e f f}\right)_{\exp }^{\text {a) }}$ & $\begin{array}{l}\left(\beta_{e f f}\right)_{\mathrm{MC}}^{\text {a) }} \text { by } \\
\text { Ratio to Exp. }\end{array}$ & $\begin{array}{l}\text { McCARD } \\
\text { RSD (\%) }\end{array}$ & $\left(\beta_{e f f} / \Lambda\right)_{\exp }^{b}$ & $\begin{array}{l}\left(\beta_{\text {eff }} / \Lambda\right)_{\mathrm{MC}}{ }^{\mathrm{b})} \\
\text { Ratio to Exp. }\end{array}$ & $\begin{array}{l}\text { McCARD } \\
\operatorname{RSD}(\%)\end{array}$ \\
\hline Godiva & - & 0.00640 & 1.01 & 0.42 & $1.11 \times 10^{6}$ & 1.03 & 0.42 \\
\hline \multirow{4}{*}{ TCA } & $1.50 \mathrm{U}$ & 0.00771 & 1.00 & 1.32 & 219 & 1.01 & 1.34 \\
\hline & $1.83 \mathrm{U}$ & 0.00760 & 1.00 & 1.38 & 201 & 0.98 & 1.39 \\
\hline & $2.48 \mathrm{U}$ & 0.00765 & 0.98 & 1.31 & 175 & 0.97 & 1.32 \\
\hline & $3.00 \mathrm{U}$ & 0.00749 & 1.02 & 1.29 & 161 & 0.98 & 1.29 \\
\hline
\end{tabular}

a) $\left(\beta_{\text {eff }}\right)_{\text {exp }}$. is the experimental $\beta_{\text {eff }}$ while $\left(\beta_{\text {eff }}\right)_{\mathrm{MC}}$ from McCARD calculations.

${ }^{b)}\left(\beta_{\text {eff }} / \Lambda\right)_{\text {exp }}$ is the experimental $\beta_{\text {eff }} / \Lambda$ while $\left(\beta_{\text {eff }} / \Lambda\right)_{\mathrm{MC}}$ from McCARD calculations. 


\section{WHOLE -CORE TRANSPORT CALCULATIONS}

The commercial nuclear reactor cores generally have higher dominance ratios (DRs) than critical assemblies and research reactor cores. A higher DR of a nuclear system implies a longer inter-generational correlation of the FSD in the $\mathrm{MC}$ eigenvalue calculation, which requires more inactive cycle runs for the FSD to converge to the fundamental mode and becomes the source of big bias of the sample variance. In order to cope with these two problems, McCARD is equipped with the on-the-fly stopping criteria of terminating inactive cycle runs automatically and various real variance estimation methods.

\subsection{Inactive Cycle Stopping Criteria}

Anterior or on-the-fly convergence criteria [15] implemented in McCARD is designed to initiate automatic change of inactive cycle MC runs to the active ones by detecting the onset of the stationary or the fundamentalmode FSD. There are two types of anterior stopping criteria distinguished by the capital letters A and B in McCARD.

The type-A criterion is characterized by the following two equations;

$\left|S_{m}^{i}-S_{m}^{i-L}\right|<\kappa \sqrt{2\left(\sigma^{2}\left[S_{m}^{i}\right]-\operatorname{cov}\left[S_{m}^{i}, S_{m}^{i-L}\right]\right)} ; m=1,2, \cdots, N_{m}$,

and

$\frac{\text { the number of cells not satisfying Eq. (1a) }}{\text { the total number of cells }}<C(\kappa)$.

$S_{m}^{t}(t=i$ or $i-L)$ is the FSD of cell $m$ at cycle $t$ defined by $S_{m}^{t}=\int_{V_{m}} S^{t}(\mathbf{r}) d \mathbf{r}$ where $S^{t}(\mathbf{r})$ is the normalized FSD of neutrons born at location $\mathbf{r}$ with any energy and direction at cycle $\mathrm{t}$ and $V_{m}\left(m=1,2, \cdots, N_{m}\right)$ the spatial volume of cell $m$ with $N_{m}$ representing the total number of non-overlapping cells comprising the whole system.

Equation (1a) of the criterion $\mathrm{A}$ is based on a rationale that, when the FSD is converged, the difference between two fission sources $L$ cycles apart, $S_{m}^{i}$ and $S_{m}^{i-L}$, must be statistically indistinguishable. Mathematically, this rationale is expressed as that the following conditions must be met for all cells.

$$
\left|\frac{S_{m}^{i}-S_{m}^{i-L}}{S_{m}^{i}}\right|<\kappa \cdot \sigma\left[\frac{S_{m}^{i}-S_{m}^{i-L}}{S_{m}^{i}}\right] ; m=1,2, \cdots, N_{m} .
$$

$\sigma$ is the SD of the bracketed quantity and $\kappa$ is an integer, say, 1 or 2 or 3. Equation (1a) is derived after a little algebraic manipulation of Eq. (2) [15]. Because of the statistical behavior of $\left(S_{m}^{i}-S_{m}^{i-L}\right)$, there may be cells whose fission sources do not satisfy Eq. (1a). Equation (1b) is augmented to take into account the presence of such cells. Actually, $C(\kappa)$ in Eq. (1b) depends on $\kappa$ as well as the distribution function of the relative differences, $\left(S_{m}^{i}-S_{m}^{i-L}\right) / S_{m}^{i}$, for all $m$. Under an assumption that $\left(S_{m}^{i}-S_{m}^{i-L}\right) / S_{m}^{i}$ follows the normal distribution,
$C(\kappa=1) \approx 32 \%, C(\kappa=2) \approx 4.6 \%$, and $C(\kappa=3) \approx 0.3 \%$.

The type $\mathrm{B}$ criterion is specified by

$$
\sum_{m=1}^{N_{m}} \frac{\left(S_{m}^{i}-S_{m}^{i-L}\right)^{2}}{2\left(\sigma^{2}\left[S_{m}^{i}\right]-\operatorname{cov}\left[S_{m}^{i}, S_{m}^{i-L}\right]\right)}<\chi^{2}\left(N_{m}, \alpha\right),
$$

where $\chi^{2}\left(N_{m}, \alpha\right)$ denotes the value $v$ that satisfies probability $\{Z>v\}=\int_{v}^{\infty} p_{z}(z) d z=\alpha$ in which $p_{z}(z)$ is the chi-square distribution of $N_{m}$ degrees of freedom. This criterion is based on an assumption that $\left(S_{m}^{i}-S_{m}^{i-L}\right) / S_{m}^{i}\left(m=1,2, \cdots, N_{m}\right)$ are a set of $N_{m}$ independent normal random variables. On this assumption, the square sum of the normalized relative differences, $Z$, given by

$$
Z=\sum_{m=1}^{N_{m}}\left\{\frac{\left(\frac{S_{m}^{i}-S_{m}^{i-L}}{S_{m}^{i}}\right)-\frac{\overline{S_{m}^{i}-S_{m}^{i-L}}}{S_{m}^{i}}}{\sigma\left[\frac{S_{m}^{i}-S_{m}^{i-L}}{S_{m}^{i}}\right]}\right\}^{2},
$$

can be regarded as the random variable that follows a chisquare distribution with $N_{m}$ degrees of freedom. This is then used to derive the stopping criterion $\mathrm{B}$ as

$$
Z<\chi^{2}\left(N_{m}, \alpha\right),
$$

which can be converted to Eq. (3) after some algebraic manipulation [15].

The application of the two stopping criteria requires estimating the values of $\sigma^{2}\left[S_{m}^{i}\right], \operatorname{cov}\left[S_{m}^{i}, S_{m}^{i-L}\right]$, and $L$. Reference 15 presents the details on how to estimate them using the cycle-by-cycle error propagation model [42].

The two stopping criteria implemented in McCARD are applied $[15,43]$ for various nuclear systems. As an illustration to demonstrate the effectiveness of the two criteria, their application results for the MC Wielandt calculations [29] of the fuel storage facility problem [44] are shown here. Table 3 shows the comparisons of the convergence cycles detected by the two-stopping criteria and the Ueki's posterior method [16] as a function of $k_{\mathrm{e}}$, a key parameter of the Wielandt eigenvalue calculation method. $k_{\mathrm{e}}=\infty$ means the normal MC eigenvalue calculations. $t_{s}$ is the cycle number at which the inactive cycle MC runs are halted by the stopping criteria and the active cycle $\mathrm{MC}$ runs are immediately activated. $t_{\mathrm{UPM}}$ denotes the cycle number signaling the onset of a stationary cycle estimated by the Ueki's posterior source convergence diagnosis. It is noteworthy to observe $t_{s}>t_{\mathrm{UPM}}$ for all the test cases. This indicates that the two criteria adequately terminate the inactive cycle runs for the MC Wielandt calculations.

The activation of the on-the-fly convergence diagnostics is an option in the McCARD input. The output file prints out the cycle number signaling the onset of the active cycle runs by on-the-fly convergence diagnostics in comparison with that of the Ueki's posterior method after the completion of the MC runs. 


\subsection{Real Variance Estimation}

It is well known that the sample variance of a tally estimate tends to be smaller than its true or real variance in the $\mathrm{MC}$ eigenvalue calculations. McCARD provides four methods available currently to estimate the real variance of a tallied parameter: the Gelbard's batch method [17], the Ueki's method [18], the FSD's inter-cycle correlation method [19], and the history-based batch method [20].

The Gelbard's batch method estimates the real variance by splitting the whole active cycles into non-overlapping batches, averaging cycle-wise tally estimates over each batch, and estimating the sample variance from the batch-averages. The method is based on the idea that the correlations between the batch-averaged estimations are

Table 3. Performance Characteristics of Anterior Stopping Criteria for the Fuel Storage Facility Problem

\begin{tabular}{c|c|c|c|c}
\hline \multirow{2}{*}{$k_{e}$} & \multirow{2}{*}{$L$} & \multirow{2}{*}{$t_{U P M}$} & \multicolumn{2}{|c}{$t_{s}$} \\
\cline { 4 - 5 } & & & Type-A $^{\mathrm{a})}$ & Type-B $^{\mathrm{b})}$ \\
\hline$\infty$ & 636 & 1298 & 1482 & 1612 \\
\hline 10.0 & 576 & 1385 & 1438 & 1632 \\
\hline 2.0 & 503 & 717 & 1048 & 1071 \\
\hline 1.5 & 266 & 571 & 687 & 719 \\
\hline 1.4 & 282 & 507 & 676 & 707 \\
\hline 1.3 & 254 & 454 & 625 & 635 \\
\hline 1.2 & 195 & 352 & 475 & 491 \\
\hline 1.1 & 174 & 342 & 427 & 430 \\
\hline 1.0 & 84 & 153 & 228 & 235 \\
\hline 0.95 & 54 & 131 & 152 & 168 \\
\hline
\end{tabular}

${ }^{\text {a) }} C(1)=31.7 \%$. ${ }^{\text {b) }} \alpha=0.9$. expected to become much weaker than those between the individual cycle values and thus the bias of the sample variance can be reduced. In order to activate the Gelbard's batch method in McCARD, a user has only to specify the batch size. The Ueki's method estimates the variance bias utilizing the lag covariances, which are directly measured from the cycle-wise tally estimations. To use this method, a user should specify the maximum lag covariance length. The FSD's inter-cycle correlation method utilizes the intercycle covariance of the FSDs which can be computed by the cycle-by-cycle stochastic error's propagation model [42]. It is more accurate than the aforementioned two methods especially for problems with high DRs but requires an appropriate regional discretization to build a fission matrix required for analysis of the error propagation model. The history-based batch method makes the most of the fact that the variance bias can disappear if the samples become independent of each other. It treats a MC eigenvalue run on $N$ active cycles with $M$ histories per cycle as the sum of $N_{B}$ runs on $N$ active cycles with $M / N_{B}$ histories per cycle.

The accuracy of the real variance estimations by the Gelbard's batch, Ueki's, and FSD's inter-cycle correlation methods for commercial reactor core problems has been reported in Ref. [19]. For the purpose of a comparison of the four methods, the weakly coupled fissile array problem [45] composed of two uranyl nitrate aqueous solution slabs of $35 \mathrm{~cm}$ in width, $69 \mathrm{~cm}$ in depth and $50 \mathrm{~cm}$ in height separated by a $30 \mathrm{~cm}$-thick concrete slab is analyzed by McCARD. The MC eigenvalue calculations are performed to determine the fission powers and their uncertainties for seven sub-regions dividing a uranyl solution slab with the same thickness of $5 \mathrm{~cm}$ based on 500 active cycles with 1,000,000 histories per cycle. Table 4 compares the relative standard deviations (RSDs) of the regional fission powers estimated from four different real variance estimation methods with the reference RSD. $\sigma_{\mathrm{REF}}, \sigma_{\mathrm{batch}}, \sigma_{\mathrm{Ueki}}, \sigma_{\mathrm{FSD}}$, and $\sigma_{\mathrm{HB}}$ in Table 4 denote the RSD estimated by a reference calculation, Gelbard's batch, Ueki's, FSD's inter-cycle correlation, and history-based

Table 4. Comparison of the Real Variance Estimates of Region-wise Fission Power Tallies

\begin{tabular}{l|c|c|c|c|c}
\hline \multicolumn{1}{c|}{ Region } & $\sigma_{\text {REF }}(95 \%$ conf. intv. $)[\%]$ & $\sigma_{\text {batch }}[\%]$ & $\sigma_{\text {Ueki }}[\%]$ & $\sigma_{\text {FSD }}[\%]$ & $\sigma_{\text {HB }}(95 \%$ conf. intv. $)[\%]$ \\
\hline 1, Outer-most & $0.546(0.490,0.619)$ & 0.106 & 0.147 & 0.437 & $0.494(0.443,0.560)$ \\
\hline 2 & $0.539(0.483,0.611)$ & 0.095 & 0.133 & 0.434 & $0.494(0.443,0.560)$ \\
\hline 3 & $0.533(0.477,0.604)$ & 0.092 & 0.131 & 0.431 & $0.490(0.439,0.555)$ \\
\hline 4 & $0.522(0.468,0.591)$ & 0.098 & 0.138 & 0.424 & $0.484(0.434,0.548)$ \\
\hline 5 & $0.506(0.453,0.573)$ & 0.105 & 0.148 & 0.413 & $0.470(0.421,0.532)$ \\
\hline 6 & $0.484(0.434,0.549)$ & 0.100 & 0.141 & 0.395 & $0.451(0.404,0.511)$ \\
\hline 7, Inner-most & $0.429(0.385,0.487)$ & 0.090 & 0.124 & 0.352 & $0.400(0.358,0.453)$ \\
\hline
\end{tabular}




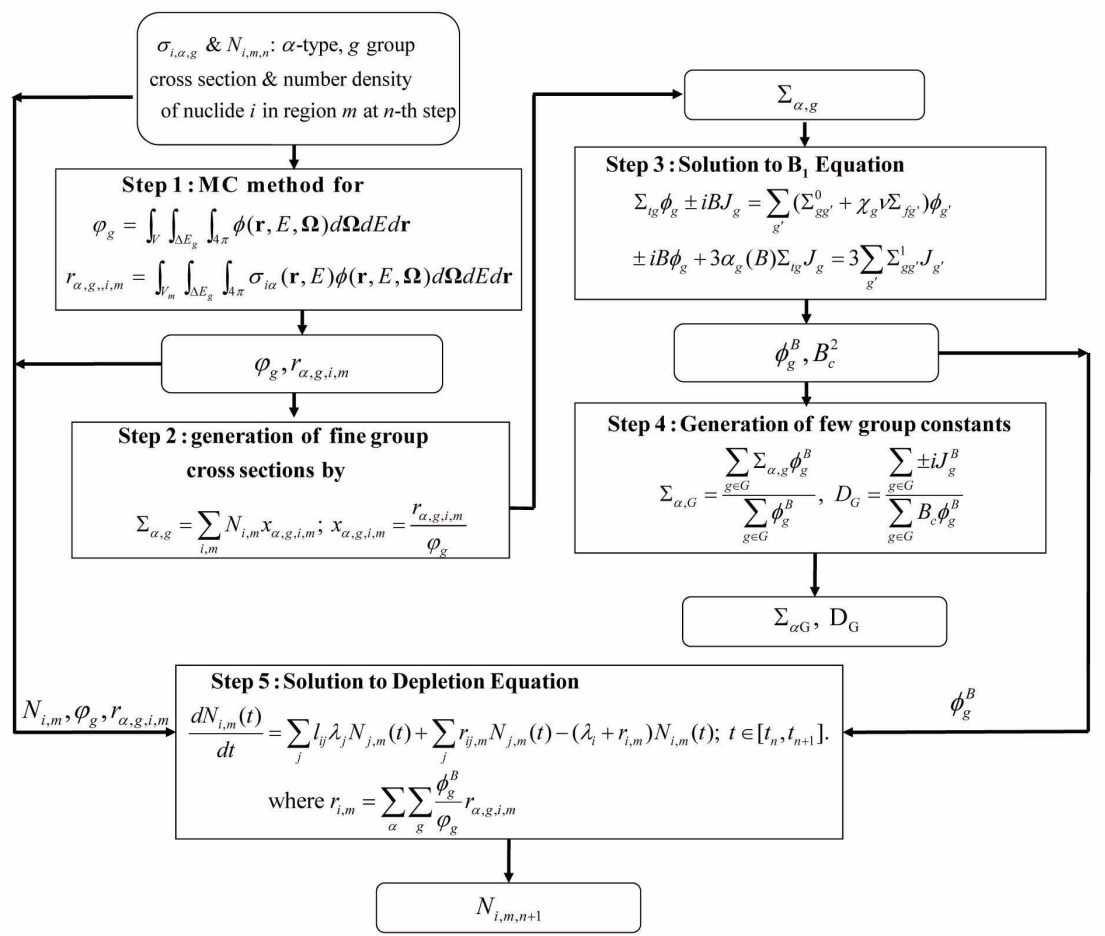

Fig. 5. Schematic Diagram for the $\mathrm{B}_{1}$ Theory-augmented MC Method

batch methods, respectively. The batch size is taken as 10 for the Gelbard's batch method while the maximum lag covariance length for the Ueki's method is assumed to be 10 . For $\sigma_{\mathrm{HB}}, N_{B}$ is set at 100 . It is noted that real variances are better predicted by the FSD's inter-cycle method and the history-based batch method than the two other methods implemented in McCARD.

\subsection{Temperature Feedback Calculations}

Like its deterministic counterpart, the MC wholecore neutronics analysis must take into account thermalhydraulic $(\mathrm{T} / \mathrm{H})$ feedback in order to determine the neutron distribution consistent with temperature distribution of the system. This is done by a repetition of the MC neutronics and $\mathrm{T} / \mathrm{H}$ calculations. Cell-averaged temperatures in the McCARD calculations are updated by a simple heat transfer model [46]. The convergence of $\mathrm{T} / \mathrm{H}$ feedback coupled with neutronics calculations is checked by monitoring the change of the cell-wise temperature,

$$
\max _{m}\left|T_{m}^{t+1}-T_{m}^{t}\right| \leq \varepsilon_{T} \text { or }\left|T_{m}^{t+1}-T_{m}^{t}\right| \leq C \sigma_{\mathrm{R}, \mathrm{est}}\left[T_{m}^{t+1}-T_{m}^{t}\right] .
$$

$m$ and $t$ are the cell index and T/H coupled iteration index, respectively. $T_{m}^{t}$ is the average temperature of cell $m$ at iteration $t . \sigma_{\mathrm{R}, \text { est }}$ is the estimated real $\mathrm{SD}$ by the methods introduced in Section 3.2. $\varepsilon_{T}$ and $C$ are user-provided convergence parameters. For example, they may be set at $5 \mathrm{~K}$ and 3 , respectively.
Coolant density corresponding to the temperature of the coolant cell is updated from a user-defined property table. Currently, the neutron cross section libraries for the updated temperature are chosen as the ones corresponding to the closest temperature from pre-prepared temperaturedependent cross section library sets. The McCARD capability of the whole-core neutronics analysis with the $\mathrm{T} / \mathrm{H}$ feedback is demonstrated in Ref. 47. Some of the details on the whole-core neutronics analysis with the $\mathrm{T} / \mathrm{H}$ feedback is available in Ref. 48.

\section{FEW GROUP DIFFUSION THEORY CONSTANT GENERATION}

McCARD can generate the few group constants by the $\mathrm{B}_{1}$ theory-augmented MC method [22, 49, 50]. As shown in Fig. 5, this method consists of a sequence of steps; step 1 for the MC computation for the infinite medium spectrum $\left(\varphi_{g}\right)$ of and microscopic reaction rates $\left(r_{\alpha, g, i, m}\right)$ in the nuclear fuel pin or the FA, step 2 for processing them into fine group reaction cross sections $\left(\Sigma_{\alpha, g}\right)$ to prepare inputs to $B_{1}$ equation, step 3 for solving $B_{1}$ equation for the critical spectrum $\left(\phi_{g}^{B}\right)$ and critical buckling $\left(B_{c}^{2}\right)$, and step 4 for generation of the critical spectrum weighted few group cross sections $\left(\Sigma_{\alpha, G}\right)$ and diffusion constants $\left(D_{G}\right)$. Steps 1 to 4 calculations are performed at a given depletion time $t_{n}(n=0,1,2, \ldots)$ and they are enough to 
Table 5. Comparison of Two Group Constants at Fresh Fuel State

\begin{tabular}{c|c|c|c|c}
\hline \multirow{2}{*}{ Code } & \multicolumn{2}{|c|}{ McCARD } & \multicolumn{2}{c}{ Relative Difference from McCARD [\%] } \\
\cline { 2 - 5 } & XS & RSD [\%] & CASMO3 & HELIOS 1.8 \\
\hline$B_{0}^{2}$ & $5.09876 \times 10^{-3}$ & - & -1.90 & -0.69 \\
\hline$D_{1}$ & $1.46317 \times 10^{0}$ & 0.12 & 3.10 & 0.16 \\
\hline$\Sigma_{a, 1}$ & $9.24314 \times 10^{-3}$ & 0.10 & 0.91 & -0.25 \\
\hline$\Sigma_{s, 12}$ & $1.48781 \times 10^{-2}$ & 0.12 & 1.67 & 0.43 \\
\hline$v \Sigma_{f, 1}$ & $7.06284 \times 10^{-3}$ & 0.08 & 2.94 & 1.22 \\
\hline$D_{2}$ & $5.00963 \times 10^{-1}$ & 0.13 & -5.61 & -0.41 \\
\hline$\Sigma_{a, 2}$ & $8.58098 \times 10^{-2}$ & 0.14 & -0.06 & -0.10 \\
\hline$v \Sigma_{f, 2}$ & $1.45452 \times 10^{-1}$ & 0.14 & -0.95 & 0.28 \\
\hline$k_{\text {inf }}$ & 1.33971 & 0.04 & -0.12 & \\
\hline
\end{tabular}

\begin{tabular}{|c|c|c|c|c|c|}
\hline 1.355 & 1.366 & 1.347 & 1.173 & 0.527 & \\
\hline 0.188 & 0.132 & 0.133 & 0.147 & 0.219 & \\
\hline-1.58 & -1.54 & -0.78 & 0.78 & 1.54 & \\
\hline 1.370 & 1.390 & 1.431 & 1.028 & 0.429 & \\
\hline 0.132 & 0.091 & 0.093 & 0.110 & 0.185 & \\
\hline-1.56 & -1.33 & -0.37 & 0.37 & 1.84 & \\
\hline 1.346 & 1.429 & 1.150 & 0.596 & \multirow{9}{*}{$\begin{array}{r}\text { Ref(McCARD) } \\
\text { cCARD/MASTER) }\end{array}$} & \\
\hline 0.133 & 0.093 & 0.104 & 0.156 & & \\
\hline-0.57 & -0.06 & 0.01 & 0.97 & & $k_{\text {eff }}$ \\
\hline 1.168 & 1.025 & 0.599 & \multirow{6}{*}{ 2Step } & & $.02907 \pm 0.00016$ \\
\hline 0.147 & 0.110 & 0.156 & & & 1.02915 \\
\hline 0.87 & 0.78 & 0.69 & & & \\
\hline 0.523 & 0.424 & & & & $P_{\text {Ref }}$ \\
\hline 0.220 & 0.185 & & & & Rel. Err. (\%) \\
\hline 2.15 & 2.81 & & & & Diff \\
\hline
\end{tabular}

$\mathrm{P}_{\text {Ref }}=$ McCARD power $\quad \mathrm{P}_{2 \text { setp }}=$ McCARD/MASTER power

Rel. Err. $(\%)=$ Relative Error of McCARD power $\quad$ Diff $=\left(P_{2 \text { step }}-P_{\text {Ref }}\right) / P_{\operatorname{Ref}^{\ngtr}} 100$

Fig. 6. The Assembly-wise Normalized Power (NP) Distribution for 3D PWR Core Problem

generate the few group constants at $t_{n}$. In order to generate those at $t_{n+1}$, however, the depletion calculation step to be discussed later in Section 5.3 needs be conducted to prepare critical spectrum corrected nuclide number densities at $t_{n+1}, N_{i, m, n+1}$, namely the nuclide number density inputs for step $1 \mathrm{MC}$ calculation at the next depletion time $t_{n+1}$. Note that McCARD method differs from some of MC methods suggested earlier [51-53] for the few group constants generation in that it makes use of $\mathrm{B}_{1}$ spectrum [54]. Note also that $B_{1}$ spectrum is used to take into account the effect of critical spectrum on the fuel depletion as well as to generate critical spectrum-weighted few group nuclear reaction cross sections and the few group diffusion constants.

Since the $\mathrm{B}_{1}$ theory-augmented $\mathrm{MC}$ method was implemented into McCARD for the first time in 2008
[22], the qualification of the few group constants from McCARD has been examined from the two aspects of their applications to neutronics design computations. One is how well they are compared with those from the commercial FA spectrum codes such as HELIOS [13] and CASMO [38]. The other is how accurately they can produce core neutronics computations for core neutronics test problems in comparison with the reference MC calculations or the neutronics design reports (NDR) or the measured plant data $[49,50]$. As an illustration, Table 5 shows a comparison of the two-group constants determined by HELIOS and CASMO, and McCARD for a 17x17 PWR FA [22]. Remarkably good agreements are observed between the two group constants from McCARD and those from HELIOS and CASMO. As the other illustrations, Figures 6 and 7 

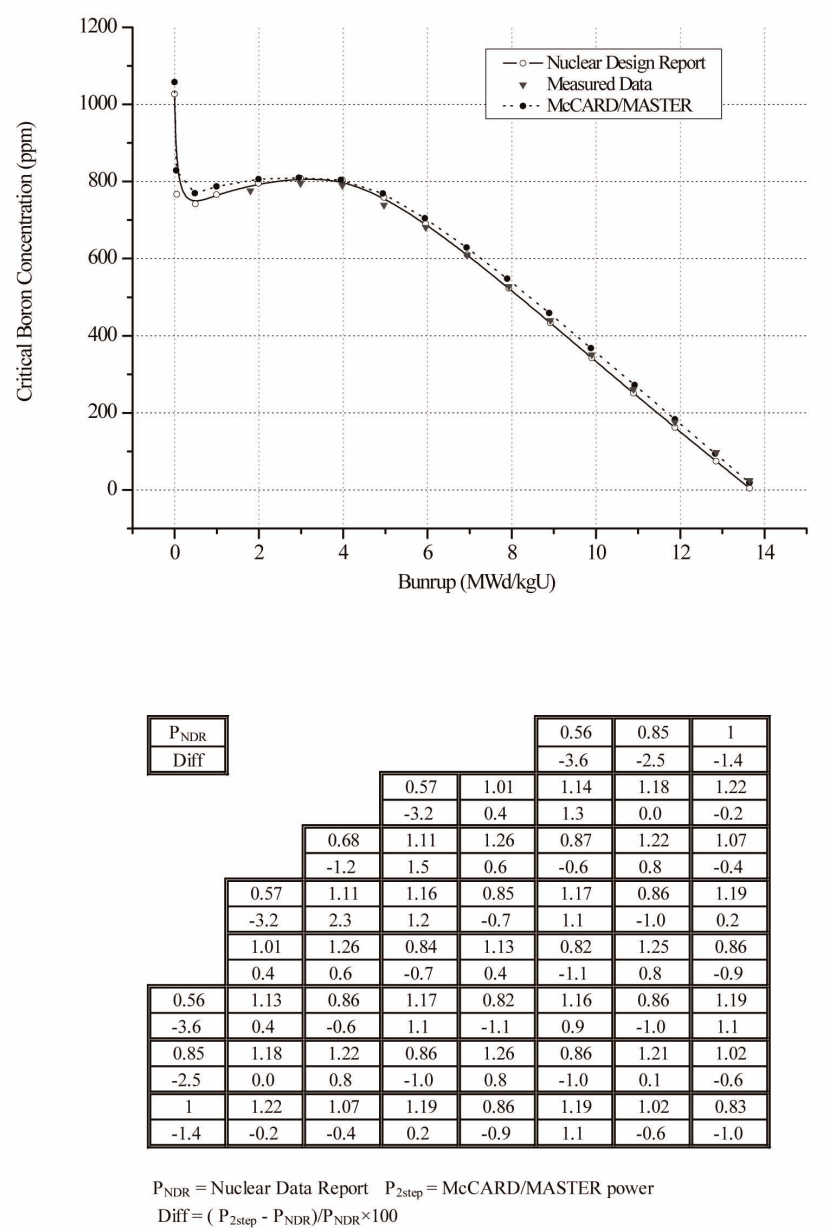

Fig. 7. (a) Critical Boron Letdown Curve and (b) Assembly-wise Normalized Power Distribution in YGN 4 Reactor Core at 13 EFPD

show the assemblywise normalized power distribution and $k_{\text {eff }}$ by two-step code system McCARD/MASTER calculations for a 3-dimensional (3D) PWR core [50] and the cycle 1 PWR core of Yonggwang unit 4 (YGN4) [55], respectively. The McCARD/MASTER calculations for the FA power distribution and $k_{\text {eff }}$ of the two PWR cores display excellent agreements with the reference McCARD calculations in case of the 3D PWR and the measurements in case of YGN4 cycle 1. Needless to mention, these are seen as warranting the quality of the few group constants from McCARD. For the sake of reference, MASTER [56] is a nodal diffusion theory core analysis code of Korea Atomic Energy Research Institute.

\section{UNCERTAINTY QUANTIFICATION}

The MC reactor calculations are bound to carry statistical uncertainties with them. In addition, uncertainties of the input data may amplify those of the resulting MC calculations when the nuclear cross section and nuclide number density inputs to the $\mathrm{MC}$ calculation have uncertainties. Therefore, it is essential for MC users to be able to quantify both the statistical and the input data uncertainties of $\mathrm{MC}$ reactor calculations -preferably separately, in order to report the confidence interval of the MC results, to identify the most significant contributing input data and find ways to improve them, or to provide a quantitative basis for assessing the feasibility of the next-generation fuel or reactor system designs. McCARD has an uncertainty quantification module designed to quantify uncertainties associated with such problems as (i) criticality benchmark analysis, (ii) actinide fuel incineration analysis, and (iii) generation of fuel assembly homogenized few group cross section by the $\mathrm{MC}$ method. The problems (ii) and (iii) require composite analyses in which MC results are combined with deterministic calculations. These are so-called uncertainty propagation problems in which the uncertainties of MC calculations 
affect those of the subsequent deterministic calculations. The special feature of the uncertainty quantification module of McCARD is its ability to quantify separately the statistical uncertainty and contributions of input data uncertainties to the overall uncertainty of any reactor parameter estimated from it. The following includes a brief description on the mathematical basis of this special feature and how to handle the uncertainty propagation within the framework of the mathematical basics of uncertainty quantification.

\subsection{Mathematical Basis of McCARD Uncertainty Quantification}

Suppose that one is interested in computing a reactor parameter such as $k_{\text {eff, }}$ power densities etc. by McCARD and that the nuclear cross section and the nuclide number density input data to McCARD have uncertainties. Representing the reactor parameter of interest by $Q$ and the uncertain input data by a set of variables, $u_{1}, \cdots, u_{\ell}, \cdots, u_{L}$, one may have a functional relation between the uncertain input data and the output from them by

$$
Q_{\kappa}=Q\left(u_{1}^{\kappa}, \cdots, u_{\ell}^{\kappa}, \cdots, u_{L}^{\kappa}\right) .
$$

$\kappa$ is the index for the different input data sets that one may sample from the probability distribution function of each member of the input data and the output from it. From Eq. (7), one can formally obtain the mean value of $Q, \bar{Q}$, and the variance, $\sigma^{2}(Q)$, by

$$
\bar{Q}=\lim _{K \rightarrow \infty} \frac{1}{K} \sum_{\kappa=1}^{K} Q_{\kappa} \text { and } \sigma^{2}(Q)=\lim _{K \rightarrow \infty} \frac{1}{K-1} \sum_{\kappa=1}^{K}\left(Q_{\kappa}-\bar{Q}\right)^{2}
$$

One may compute $\bar{Q}$ and $\sigma^{2}(Q)$, a measure of uncertainty of the MC estimate $Q$, using Eq. (8) directly by obtaining $Q_{\kappa}$ for a finite sampling of input data sets, say, $K=50,100$, and the like and approximating the infinite sum by a finite sum. Though observed in Ref. 57 and 58, this approach has a couple of drawbacks. It is not only time-consuming but also lacks in information on contribution of statistical uncertainties and contributions from individual input data uncertainties to $\sigma^{2}(Q)$.

McCARD avoids these drawbacks of the direct sampling method by computing separately the two contributions to $\sigma^{2}(Q)$ by

$$
\begin{gathered}
\sigma_{S}^{2}(Q)=\lim _{K \rightarrow \infty} \frac{1}{K-1} \sum_{K=1}^{K}\left(Q_{\kappa}-<Q_{\kappa}>\right)^{2} \\
\sigma_{\mathrm{I}}^{2}(Q)=\lim _{K \rightarrow \infty} \frac{1}{K-1} \sum_{\kappa=1}^{K}\left(<Q_{\kappa}>-\bar{Q}\right)^{2} \\
=\left.\left.\sum_{\ell=1}^{L} \sum_{p=1}^{L} \operatorname{cov}\left[u_{\ell}, u_{p}\right]\left(\frac{\partial<Q>}{\partial u_{\ell}}\right)\right|_{0}\left(\frac{\partial<Q>}{\partial u_{p}}\right)\right|_{0} .
\end{gathered}
$$

Note that $Q_{\kappa}-\bar{Q}$ in Eq. (8) is rewritten as $Q_{\kappa}-<Q_{\kappa}>+<$ $Q_{\kappa}>-\bar{Q}$ which makes it split $\sigma^{2}(Q)$ into $\sigma_{\mathrm{S}}^{2}(Q)$ and $\sigma_{\mathrm{I}}^{2}(Q)$ denoting the contributions from the statistical and input data uncertainties, respectively. Note also that Eq. (10) is obtained from the first-order Taylor expansion for $<Q_{\kappa}>$ $-\bar{Q}$,

$$
<Q_{\kappa}>-\left.\bar{Q} \approx \sum_{\ell=1}^{L} \frac{\partial<Q>}{\partial u_{\ell}}\right|_{0} \cdot\left(u_{\ell}^{\kappa}-\overline{u_{\ell}}\right) .
$$

The angular bracket implies the expected value of a quantity in it, while the bar sign the mean value of a quantity under it. Thus $Q_{\kappa}-<Q_{\kappa}>$ is the statistical fluctuation of $Q_{\kappa}$ about its expected value while $u_{\ell}^{\kappa}-\bar{u}_{\ell}$ the uncertainty of the $\ell$-th member of input data, $u_{\ell}^{\kappa}$, about its mean, $\bar{u}_{\ell}$. The symbol “ $\left.\frac{\partial<\mathrm{Q}>}{\partial u_{\ell}}\right|_{0}$ implies

$$
\left.\frac{\partial<\mathrm{Q}>}{\partial u_{\ell}}\right|_{0}=\left.\frac{\partial<\mathrm{Q}\left(u_{l}^{\kappa}, \cdot \cdot, u_{\ell}^{\kappa}, \cdot \cdot, u_{L}^{\kappa}>\right.}{\partial u_{\ell}^{\kappa}}\right|_{u_{l}^{\kappa}=\overline{u_{1}}, \cdot, u_{\ell}^{\kappa}=\overline{u_{\ell}}, \cdots, u_{L}^{\kappa}=\overline{L_{L}}} .
$$

McCARD determines $\bar{Q}, \sigma_{\mathrm{S}}^{2}(Q)$, and $\left.\frac{\partial<\mathrm{Q}>}{\partial u_{\ell}}\right|_{0}$ in the
ove equations by approximating them by

$$
\begin{gathered}
\bar{Q} \approx<Q\left(\overline{u_{1}}, \cdots, \overline{u_{\ell}}, \cdots, \overline{u_{L}}\right)>, \\
\sigma_{\mathrm{S}}^{2}(Q) \approx<Q^{2}\left(\overline{u_{1}}, \cdots, \overline{u_{\ell}}, \cdots, \overline{u_{L}}\right)>-<Q\left(\overline{u_{1}}, \cdots, \overline{u_{\ell}}, \cdots, \overline{u_{L}}\right)>^{2} \\
\left.\frac{\partial<Q>}{\partial u_{\ell}}\right|_{0} \approx \frac{<Q\left(\overline{u_{1}}, \cdots, \overline{u_{\ell}}+\sigma_{\ell}, \cdots, \overline{u_{L}}\right)>-<Q\left(\overline{u_{1}}, \cdots, \overline{u_{\ell}}, \cdots, \overline{u_{L}}\right)>}{\sigma_{\ell}} \\
=\frac{\delta<Q>}{\sigma_{\ell}} .
\end{gathered}
$$

These approximations require one McCARD run with the mean value input data set, $\bar{u}_{1}, \cdots, \bar{u}_{\ell}, \cdots, \bar{u}_{L} . \delta<\mathrm{Q}>$ may be computed by a direct subtraction or MC perturbation computation. $\sigma_{\ell}$ is the SD of $\bar{u}_{\ell}$.

\subsection{S/U Analysis of Criticality Benchmark Problems}

The capability of McCARD to isolate the contribution of the input data uncertainties to the uncertainty of a MC estimate on any neutronics parameter has useful applications. For example, McCARD can estimate the effects of the nuclear cross section uncertainties on the uncertainty of $k_{\text {eff }}$ of a nuclear reactor system by the type of the cross section and by the type of the nuclide. On the assumption that input data uncertainties come from the nuclear cross sections only and that there are no correlation between cross sections of the different isotopes, $\sigma_{\mathrm{I}}^{2}\left(k_{\text {eff }}\right)$ is derived from Eq. (10) as

$$
\sigma_{\mathrm{I}}^{2}\left(k_{\mathrm{eff}}\right)=\left.\left.\sum_{i} \sum_{\alpha, g} \sum_{\alpha^{\prime}, g^{\prime}} \operatorname{cov}\left[\sigma_{\alpha, g, i}, \sigma_{\alpha^{\prime}, g^{\prime}, i}\right] \frac{\partial k_{\mathrm{eff}}}{\partial \sigma_{\alpha, g, i}}\right|_{0} \frac{\partial k_{\mathrm{eff}}}{\partial \sigma_{\alpha^{\prime}, g^{\prime}, i}}\right|_{0}
$$

where $\sigma_{\alpha, g, i}$ is the $\alpha$-type cross section of nuclide $i$ for 
Table 6. Contribution of ${ }^{235} \mathrm{U}$ Cross Section Uncertainties to $o\left(k_{\text {eff }}\right)$ of the Godiva Critical Assembly by the Type of the Nuclear Data

\begin{tabular}{|c|c|c|c|}
\hline Code & $\begin{array}{l}\text { Neutronics } \\
\mathrm{S} / \mathrm{U}\end{array}$ & $\begin{array}{l}\text { ANISN } \\
\text { SUSD3D }\end{array}$ & $\begin{array}{l}\text { McCARD } \\
\text { McCARD }\end{array}$ \\
\hline \multicolumn{2}{|c|}{$\begin{array}{l}\text { Energy Group } \\
\text { Covariance Data }\end{array}$} & $\begin{array}{l}44 \text { groups } \\
44 \text { groups }\end{array}$ & $\begin{array}{l}\text { Continuous } \\
44 \text { groups }\end{array}$ \\
\hline \multirow{8}{*}{$\sigma\left(k_{e f f}\right)(\%)$} & \multirow{4}{*}{$\begin{array}{c}v, v \\
(\mathrm{n}, \gamma),(\mathrm{n}, \gamma) \\
(\mathrm{n}, \gamma),(\mathrm{n}, \mathrm{n}) \\
(\mathrm{n}, 2 \mathrm{n}),(\mathrm{n}, 2 \mathrm{n})\end{array}$} & 0.15 & 0.15 \\
\hline & & 0.17 & 0.16 \\
\hline & & 0.05 & 0.05 \\
\hline & & 0.01 & 0.01 \\
\hline & \multirow{2}{*}{$\begin{array}{c}(\mathrm{n}, \mathrm{fis}),(\mathrm{n}, \mathrm{fis}) \\
(\mathrm{n}, \mathrm{n}),(\mathrm{n}, \mathrm{n})\end{array}$} & 0.17 & 0.17 \\
\hline & & 0.31 & 0.33 \\
\hline & \multirow[t]{2}{*}{$(\mathrm{n}, \mathrm{n}),\left(\mathrm{n}, \mathrm{n}^{\prime}\right)$} & $-0.44^{*}$ & $-0.46^{*}$ \\
\hline & & 0.69 & 0.69 \\
\hline \multicolumn{2}{|r|}{ total } & 0.43 & 0.47 \\
\hline
\end{tabular}

${ }^{*}$ The minus sign implies negative contribution to $\sigma^{2}\left(k_{e f f}\right)$

group $g$ neutrons. Table 6 compares McCARD results [59] for contributions of the cross section data uncertainties of ${ }^{235} \mathrm{U}$ on $\sigma_{\mathrm{I}}^{2}\left(k_{e f f}\right)$ of Godiva critical facility with those of SUSD3D [60]. The derivatives are computed using the perturbation calculation routine of McCARD in which the perturbation effect on the free-flight and collision kernels are estimated by the higher-order differential operator sampling method [61] while that on the FSD is taken into account either by the fission source perturbation (FSD) method [62] or the adjoint-weighted perturbation (AWP) method [23]. Figure 8 shows the $k$ uncertainties generated from the JENDL covariance data for the ICSBEP benchmarks.

\subsection{Uncertainty Propagation in McCARD Burnup Analysis}

McCARD conducts the MC burnup analysis using the built-in subroutine program providing the solution to the Bateman equations;

$$
\begin{array}{r}
\frac{d N_{i, m}(t)}{d t}=\sum_{j} l_{i j} \lambda_{j} N_{j, m}(t)+\sum_{j} r_{i j, m} N_{j, m}(t) \\
-\left(\lambda_{i}+r_{i, m}\right) N_{i, m}(t), t \in\left[t_{n}, t_{n+1}\right], \\
N_{i, m}\left(t_{n}\right)=N_{i, m, n},
\end{array}
$$

where

$$
r_{i j, m}=\sum_{\alpha} \sum_{g} f_{i j, \alpha} \omega_{g} r_{\alpha, g, i, m}, r_{i, m}=\sum_{j} r_{j i, m} ; \omega_{g}=\frac{\phi_{g}^{B}}{\varphi_{g}},
$$

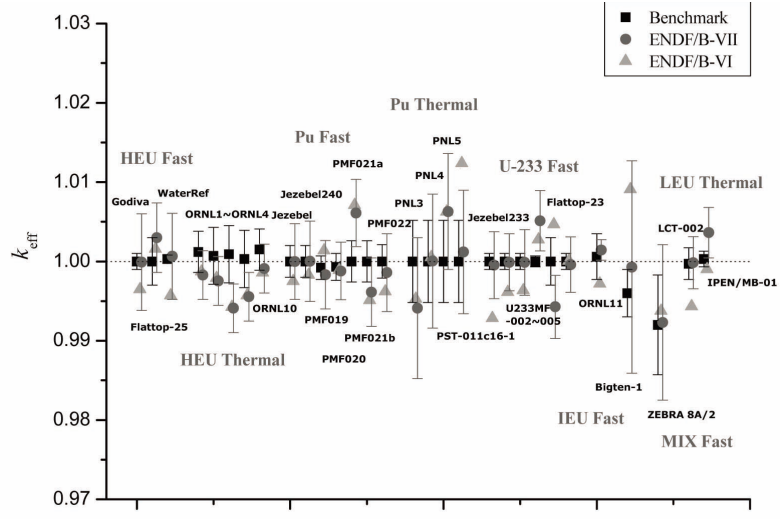

Fig. 8. McCARD Estimates on $k_{e f f}$ and $\sigma\left(k_{e f f}\right)$ for ICSBEP Benchmark Problems

$N_{\ell, m}(t)(\ell=i, j)$ is the number density of nuclide $\ell$ in cell $m$ at time $t . r_{i j, m}$ is the critical spectrum corrected microscopic reaction rate of nuclide $j$ in region $m$ leading to creation of nuclide $i . f_{i j, \alpha}$ is the expected number of nuclide $i$ formed when an $\alpha$-type reaction of nuclide $j$ takes place. $\omega_{g}$ is the critical spectrum correction factor defined by the ratio of the critical spectrum to the infinite spectrum. Other notations are standard. The solution to Eq. (17) is obtained first by determining MC estimates on $r_{\alpha, g, i, m}$ at every beginning of the depletion time step (DTS) $n,\left[t_{n}, t_{n+1}\right]\left(n=0,1,2, \cdots, n_{F}\right)$ by the $\mathrm{MC}$ run like step $1 \mathrm{MC}$ calculation in Fig. 5 and then by calling the depletion subroutine to determine $N_{i, m, n+1}$, the number density of nuclide $i$ in cell $m$ at $t=t_{n+1}$, for all $i$ using the initial condition $N_{i, m}\left(t=t_{n}\right)=N_{i, m, n}$. The above-described two-step MC burnup calculations which utilize the infinite medium spectrum weighted $r_{i j, m}$ with $\omega_{g}=1$ are the general practice of the MC burnup analyses today. In order to take into account the critical spectrum effect on the fuel depletion in reactors operating at the critical state, however, McCARD offers another MC depletion analysis option based on the critical spectrum corrected $r_{i j, m}$ in which $\omega_{g}$ is determined by the $\mathrm{B}_{1}$ critical spectrum $\left(\phi_{g}^{B}\right)$ from step 2 and 3 calculations in Fig. 5.

Because more than two-step calculations every DTS are performed repeatedly from the first to the last DTS to determine the composition or the nuclide number densities of the burnt fuel or FA, the cross section data uncertainties plus statistical uncertainties of parameters $r_{i j, m}$ of the first step MC calculation propagate to uncertainties of the nuclide number densities of the ensuing DTS and eventually those of the isotopic contents of the irradiated fuel. The mathematical basis for McCARD to quantify propagating uncertainties like this in $\mathrm{MC}$ depletion calculations without critical spectrum correction, namely the case of $\omega_{g}=1$ is well documented in reference 25 . Its application results for the incineration behavior of minor actinides like ${ }^{241} \mathrm{Am}$, ${ }^{243} \mathrm{Am}$, and ${ }^{244} \mathrm{Cm}$ in an Actinide FA designed for the HYPER subcritical incinerator and the SDs of the number 
Table 7. Fuel Assembly Homogenized Two Group Cross Sections and Their Percentile Relative Errors

\begin{tabular}{c|c|c|c|c|c|c}
\hline EFPD & $\Sigma_{f, 1} \pm \% \mathrm{RE}$ & $\Sigma_{f, 2} \pm \% \mathrm{RE}$ & $\Sigma_{\gamma, 1} \pm \% \mathrm{RE}$ & $\Sigma_{\gamma, 2} \pm \% \mathrm{RE}$ & $D_{1} \pm \% \mathrm{RE}$ & $D_{2} \pm \% \mathrm{RE}$ \\
\hline 0 & $2.72 \times 10^{-3} \pm 0.27$ & $6.29 \times 10^{-2} \pm 0.48$ & $6.49 \times 10^{-3} \pm 1.61$ & $4.27 \times 10^{-2} \pm 0.68$ & $1.48 \times 10^{0} \pm 2.28$ & $4.66 \times 10^{-1} \pm 0.51$ \\
\hline 7.3 & $2.71 \times 10^{-3} \pm 0.28$ & $6.24 \times 10^{-2} \pm 0.42$ & $6.50 \times 10^{-3} \pm 1.65$ & $4.41 \times 10^{-2} \pm 0.61$ & $1.48 \times 10^{0} \pm 2.33$ & $4.66 \times 10^{-1} \pm 0.48$ \\
\hline 24 & $2.71 \times 10^{-3} \pm 0.29$ & $6.18 \times 10^{-2} \pm 0.50$ & $6.53 \times 10^{-3} \pm 1.59$ & $4.56 \times 10^{-2} \pm 0.71$ & $1.48 \times 10^{0} \pm 2.29$ & $4.67 \times 10^{-1} \pm 0.50$ \\
\hline 48 & $2.70 \times 10^{-3} \pm 0.26$ & $6.17 \times 10^{-2} \pm 0.49$ & $6.55 \times 10^{-3} \pm 1.63$ & $4.63 \times 10^{-2} \pm 0.71$ & $1.48 \times 10^{0} \pm 2.28$ & $4.68 \times 10^{-1} \pm 0.54$ \\
\hline 96 & $2.67 \times 10^{-3} \pm 0.30$ & $6.15 \times 10^{-2} \pm 0.50$ & $6.58 \times 10^{-3} \pm 1.61$ & $4.74 \times 10^{-2} \pm 0.71$ & $1.48 \times 10^{0} \pm 2.36$ & $4.68 \times 10^{-1} \pm 0.53$ \\
\hline 145 & $2.65 \times 10^{-3} \pm 0.30$ & $6.14 \times 10^{-2} \pm 0.60$ & $6.60 \times 10^{-3} \pm 1.67$ & $4.82 \times 10^{-2} \pm 0.78$ & $1.48 \times 10^{0} \pm 2.30$ & $4.67 \times 10^{-1} \pm 0.55$ \\
\hline 193 & $2.63 \times 10^{-3} \pm 0.39$ & $6.13 \times 10^{-2} \pm 0.61$ & $6.65 \times 10^{-3} \pm 1.49$ & $4.88 \times 10^{-2} \pm 0.73$ & $1.48 \times 10^{0} \pm 2.31$ & $4.66 \times 10^{-1} \pm 0.53$ \\
\hline 242 & $2.60 \times 10^{-3} \pm 0.40$ & $6.11 \times 10^{-2} \pm 0.72$ & $6.67 \times 10^{-3} \pm 1.63$ & $4.95 \times 10^{-2} \pm 0.78$ & $1.49 \times 10^{0} \pm 2.33$ & $4.65 \times 10^{-1} \pm 0.53$ \\
\hline 290 & $2.58 \times 10^{-3} \pm 0.43$ & $6.09 \times 10^{-2} \pm 0.78$ & $6.68 \times 10^{-3} \pm 1.65$ & $5.01 \times 10^{-2} \pm 0.79$ & $1.49 \times 10^{0} \pm 2.30$ & $4.64 \times 10^{-1} \pm 0.51$ \\
\hline 339 & $2.56 \times 10^{-3} \pm 0.50$ & $6.07 \times 10^{-2} \pm 0.92$ & $6.71 \times 10^{-3} \pm 1.63$ & $5.06 \times 10^{-2} \pm 0.89$ & $1.49 \times 10^{0} \pm 2.34$ & $4.63 \times 10^{-1} \pm 0.55$ \\
\hline
\end{tabular}

density estimates of the three nuclides with burnup are also presented there along with discussions on the implication of the uncertainty quantification as a means to assess the design feasibility of incinerators of minor Actinides like HYPER. The McCARD option to quantify uncertainties of the critical spectrum corrected fuel depletion calculations is a relatively recent addition. The formulation for this option can be made in exactly same way as the infinite spectrum weighted counterpart by using the basic equations in Section 5.1. Figure 9 shows critical spectrum corrected McCARD depletion calculations for ${ }^{235} \mathrm{U}$ burnup in a 17x17 PWR FA in comparison with the infinite medium weighted counterpart. The two calculations did not show the noticeable differences in the number density estimates of ${ }^{235} \mathrm{U}$ but they do in the uncertainty estimates of number densities as displayed in the inset of Fig. 9.

\subsection{Uncertainty Quantification of Few Group Constants}

McCARD generates the few group constants through multi-step calculations in sequence as discussed in Section 4. Because a sequence of steps are involved and outputs of any one step become inputs of the ensuing step, the uncertainties of the nuclear cross section and nuclide number density input data to step $1 \mathrm{MC}$ calculation propagates to uncertainties of step $1 \mathrm{MC}$ outputs and to those of the ensuing step outputs and finally to those of few group constants. The McCARD module to quantify the uncertainties of few group constants is as recent as its few group constants generation module. The mathematical formulation for this module can be derived in the similar way as that for uncertainty propagation analysis in McCARD depletion analysis and detailed description on

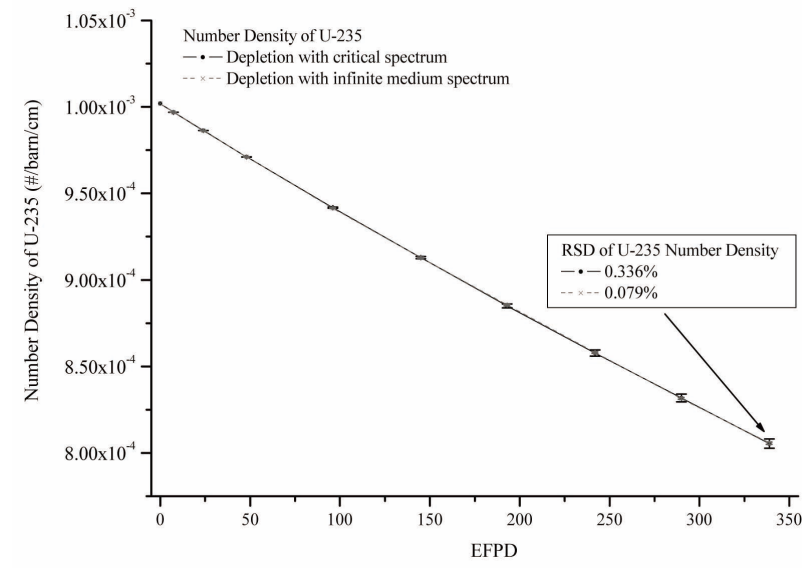

Fig. 9. Critical Spectrum Corrected McCARD Depletion Calculations for ${ }^{235} \mathrm{U}$ Burnup in a 17x17 PWR FA

it will be presented elsewhere. Table 7 shows homogenized two group constants and their percentile relative errors (\% RE) of the 17x17 PWR FA with burnup to demonstrate the capability of McCARD to quantify the uncertainties of the few group constants. The necessary covariance data files for this demonstration are from JENDL 3.3. Only covariance data files of the two major uranium isotopes ${ }^{235} \mathrm{U}$ and ${ }^{238} \mathrm{U}$ are used.

\section{CONCUSIONS}

McCARD is a specialized and highly efficient MC code for neutronics analyses of nuclear reactors and fuel 
systems, because it is developed exclusively for those purposes. Its burnup analysis capability based on precise geometric modeling and efficient load balancing makes it a very useful MC code for nuclear design and analysis of nuclear reactors - particularly research and Gen-IV reactors. Its capabilities of the few group constants generation and uncertainty quantification offer wide-range applications to reactor calculations.

McCARD is equipped with a great many state-of-theart technologies aimed at the $\mathrm{MC}$ whole core calculations. Yet it is still in evolutionary process. To make it a more versatile and user-friendly MC code, more works and research efforts should be made in order to improve and expand its computational modules, to extend the area of its applications beyond neutronics analysis of the currentgeneration fission reactors, to incorporate an advanced scheme to handle temperature-dependent cross section data, and to strengthen the visual representation of I/O through advanced graphic user interface and CAD interfacing module. The results of these efforts will be reported.

\section{REFERENCES}

[ 1 ] H. J. Shim, C. H. Kim, W. S. Park, H. K. Joo, "Monte Carlo Depletion Analysis of a PWR with the MCNAP," Proc. Int. Conf. On Mathematics and Computation, $M \& C^{\prime} 99$, Madrid, Spain (1999).

[2] A. G. Croff, "A User's Manual for ORIGEN2 Computer Code," ORNL/TM-7175, Oak Ridge National Laboratory (1980).

[ 3 ] M. H. Chang, et al., "Basic Design Report of SMART," KAERI/TR-2142/2002, Korea Atomic Energy Research Institute (2002).

[4] H. C. Lee, C. K. Jo, H. J. Shim, Y. H. Kim, and J. M. Noh, "Decay Heat Analysis of VHTR Cores by Monte Carlo Core Depletion Calculation," Ann. Nucl. Eng., 37, 1356 (2010).

[5] D. H. Hahn, et al., "KALIMER-600 Conceptual Design Report,” KAERI/TR-3381/2007, Korea Atomic Energy Research Institute (2007).

[ 6 ] Chang Hyo Kim, et al., "Neutronics Design and Performance Analysis of Korean ITER TBM by Monte Carlo Method," KAERI/CM-875/2005, Korea Atomic Energy Research Institute (2005).

[ 7 ] W. S. Park, T. Y. Song, B. O. Lee, C. K. Park, "A Preliminary design study for the HYPER System," Nucl. Eng. Design, 219, 207 (2002).

[8] V. Bournos, et al., "YALINA-Booster Benchmark Specifications for the IAEA Coordinated Research Projects on Analytical and Experimental Benchmark Analysis on Accelerator Driven Systems, and Low Enriched Uranium Fuel Utilization in Accelerator Driven Sub-Critical Assembly Systems," IAEA (2007).

[9] J. R. Maiorino and U. D. Bitelli, "Specification of the sub critical core of the IPEN/MB-01 driven by a neutron generator within the IAEA sub CRP on Low Enrichment Uranium(LEU) Fuel Utilization in Accelerator Driven Sub Critical Assembly System(ADS), Phase II: Sub Critical Core with control rods, one source point Rev 0," IAEA Collaborative Work on utilization of LEU in ADS, IAEA
(2007).

[10] R. E. MacFarlane and D. W. Muir, "NJOY99.0 Code System for Producing Pointwise and Multigroup Neutron and Photon Cross Sections from ENDF/B Data," PSR-480/NJOY99.0, Los Alamos National Laboratory (2000).

[11] M. Lutz and D. Ascher, "Learning Python," O'Reilly \& Associates, Inc., Sebastopol, CA (1999)

[12] S. J. Ball and R. K. Adams, "MATEXP, A General Purpose Digital Computer Program for Solving Ordinary Differential Equations by the Matrix Exponential Method," ORNL/TM1933 (1967).

[13] R. J. J. Stamm'ler, "HELIOS Methods," Studsvik Scanpower (2002).

[14] H. J. Shim and C. H. Kim, "Uncertainty Analysis of VHTR Fuel Compact with Randomly Distributed Particles," Trans. Am. Nucl. Soc., 93, 953 (2005).

[15] H. J. Shim and C. H. Kim, "Stopping Criteria of Inactive Cycle Monte Carlo Calculations," Nucl. Sci. Eng., 157, 132 (2007).

[16] T. Ueki and F. B. Brown, "Stationarity Modelling and Informatics-based Diagnostics in Monte Carlo Criticality Calculations," Nucl. Sci. Eng., 149, 38 (2005).

[17] E. M. Gelbard and R. Prael, "Computation of Standard Deviations in Eigenvalue Calculations," Prog. Nucl. Energy, 24, 237 (1990).

[18] T. Ueki, T. Mori, and M. Nakagawa, "Error Estimation and their Biases in Monte Carlo Eigenvalue Calculations," Nucl. Sci. Eng., 125, 1 (1997).

[19] H. J. Shim, C. H. Kim, "Real Variance Estimation Using an Inter-Cycle Fission Source Correlation for Monte Carlo Eigenvalue Calculations," Nucl. Sci. Eng., 162, 98 (2009).

[20] H. J. Shim, Y. H. Kim, C. H. Kim "History-Based Batch Method for a Real Variance Estimation in Monte Carlo Eigenvalue Calculations," Trans. Am. Nucl. Soc., 100, 300 (2009).

[21] H. J. Shim, Y. Kim, C. H. Kim, "Estimation of AdjointWeighted Kinetics Parameters in Monte Carlo Forward Calculations," PHYSOR 2010:Advances in Reactor Physics to Power the Nuclear Renaissance, Pittsburgh, PA, USA, May 9.14, 2010 (2010).

[22] H. J. Shim, J. Y. Cho, J. S. Song, C. H. Kim, "Generation of Few Group Diffusion Theory Constants by Monte Carlo Code," Trans. Am. Nucl. Soc., 99, 343 (2008).

[23] H. J. Shim and C. H. Kim, "Adjoint Sensitivity and Uncertainty Analyses in Monte Carlo Forward Calculations," J. Nucl. Sci. Technol., 48[12], 1453-1461 (2011).

[24] H. J. Park, H. G. Joo, C. H. Kim, H. J. Shim, "Monte Carlo Depletion and Uncertainty Analyses for a TRU Fuel Assembly Using JENDL 3.3 Covariance Data," M\&C 2009International Conference on Mathematics, Computational Methods \& Reactor Physics, Saratoga Springs, NY, May 3-7 (2009).

[25] H. J. Park, H. J. Shim and C. H. Kim, "Uncertainty Propagation in Monte Carlo Depletion Analysis," Nucl. Sci. Eng., 167, 196 (2011).

[26] H. J. Park, H. J. Shim, H. G. Joo, C. H. Kim, "Uncertainty Quantification of Few Group Diffusion Theory Constants from the B1 Theory-Augmented Monte Carlo Method," Trans. Am. Nucl. Soc., 105, 466 (2011).

[27] I. lux and L. Koblinger, "Monte Carlo Particle Transport Methods: Neutron and Photon Calculations," CRC Press, 
Boca Raton, FL (1991)

[28] J. Lieberoth, "A Monte Carlo Technique to Solve the Static Eigenvalue Problem of the Boltzmann Transport Equation," Nukleonik, 11, 213 (1968).

[29] T. Yamamoto and Y. Miyoshi, "Reliable Method for Fission Source Convergence of Monte Carlo Criticality Calculation with Wielandt's Method," J. Nucl. Sci. Technol., 41[2], 99 (2004).

[30] Peter S. Pacheco, "Prallel Programming with MPI," Morgan Kaufmann Publishers, Inc. (1997).

[31] Chang Hyo Kim, et al., "Efficiency Enhancement of Monte Carlo Calculations,” KAERI/CM-631/2002, Korea Atomic Energy Research Institute (2002).

[32] "International Handbook of Evaluated Criticality Safety Benchmark Experiments," NEA/NSC/DOC(95)03, OECD Nuclear Energy (2006).

[33] P. O. Morakinyo, M. L. Williams, F. B. K. Kam, "Analysis of the VENUS PWR Engineering Mockup Experiment Phase I: Source Distribution," NUREG/CR-3888, U.S. Nuclear Regulatory Commission, Washington, D.C. (1984).

[34] P. D'hondt, K. Van der Meer, "VIP-PWR Phase I," VIP-P 92/10 (1992).

[35] B. C. Na, "Benchmark on the VENUS-2 MOX Core Measurements," OECD/NEA report, NEA/NSC/DOC (2000)7, ISBN 92-64-18276-4 (2000).

[36] A. Yamamoto, T. Ikehara, T. Ito, and E. Saji, "Benchmark Problem Suite for Reactor Physics Study of LWR Next Generation Fuels," J. Nucl. Sci. Technol., 39[8], 900 (2002).

[37] C. H. Kim, H. J. Shim, B. S. Han, et al., "Validation of SMART-P Core Design by Monet Carlo Depletion Code," KAERI/CM-893/2005, Korea Atomic Energy Research Institute (2005).

[38] M. Edenius, B. H. Forrsen, "CASMO-3 A Fuel Assembly Burnup Program User's Manual," Studsvik/NFA-89-3, Rev. 2, Studsvik AB (1992).

[39] K. Okumura, T. Mori, M. Nakagawa and K. Kaneko, "Validation of a Continuous-Energy Monte Carlo Burn-up Code MVP-BURN and Its Application to Analysis of Post Irradiation Experiment", J. Nucl. Sci. Technol., 37[2], 128 (2000).

[40] T. Suzaki, K. Sakurai, K. Nakajima, et al., "Precise Determination of eff for Water-moderated U and U-Pu Cores by a Method Using Buckling Coefficient of Reactivity," Proc. Sixth Int. Conf. on Nuclear Criticality Safety (ICNC'99), Versailles, France, Sept. 20-24 (1999).

[41] H. Tsuruta, I. Kobayashi, T. Suzaki, et al., "Critical Sizes of Light Water Moderated UO2 and PuO2-UO2 Lattices," JAERI 1254, JAERI (1978).

[42] E. M. Gelbard and R. E. Prael, "Monte Carlo Work at Argonne National Laboratory," ANL-75-2 (NEACRP-L118), p. 202, Argonne National Laboratory (1974).

[43] H. J. Shim and C. H. Kim, "Application of Inactive Cycle Stopping Criteria for Monte Carlo Wielandt Calculations," M\&C 2009-International Conference on Mathematics, Computational Methods \& Reactor Physics, Saratoga Springs, NY, May 3-7 (2009)

[44] R. N. Blomquist and A. Nouri, "The OECD/NEA Source Convergence Benchmark Program," Trans. Am. Nucl. Soc., 87, 143 (2002).

[45] T. Yamamoto, T. Nakamura, Y. Miyoshi, "Fission Source Convergence of Monte Carlo Criticality Calculations in
Weakly Coupled Fissile Arrays," J. Nucl. Sci. Technol., 37[1], 41 (2000).

[46] H. J. Shim, B. S. Han, and C. H. Kim, "Numerical Experiment on Variance Biases and Monte Carlo Neutronics Analysis with Thermal Hydraulic Feedback," International Conference on Supercomputing in Nuclear Applications, SNA' 2003 (2003).

[47] H. G. Joo, et al., "Consistent Comparison of Monte Carlo and Whole-Core Transport Solutions for Cores with Thermal Feedback," PHYSOR 2004-The Physics of Fuel Cycles and Advanced Nuclear Systems: Global Developments, Chicago, IL, April 25-29 (2004).

[48] D. P. Weber, T. Sofu, W. S. Yang, et al., "High Fidelity LWR Analysis with the Numerical Nuclear Reactor," Nucl. Sci. Eng., 155, 395 (2007).

[49] H. J. Park, H. G. Joo, H. J. Shim, and C. H, Kim, "Assembly Depletion with Critical Spectrum in McCARD Monte Carlo Calculations and Comparison with HELIOS," PHYSOR 2010-Advances in Reactor Physics to Power the Nuclear Renaissance, Pittsburgh, USA, May 12-14 (2010).

[50] H. J. Park, H. J. Shim, H. G. Joo, C. H. Kim, "Qualification Test of Few Group Constants Generated from an MC Method by the Two-Step Neutronics Analysis System McCARD/MASTER," M\&C 2011- International Conference on Mathematics and Computational Methods Applied to Nuclear Science and Engineering, Rio de Janeiro, RJ, Brazil, May 8-12 (2011).

[51] G. Ilas and F. Rahnema, "A Monte Carlo based Nodal Diffusion Model for Criticality Analysis of Spent Fuel Storage Lattice," Ann. Nucl. Eng., 30, 1089 (2003).

[52] M. Tohjoh, M. Watananbe, and A. Yamamoto, "Application of continuous-energy Monte Carlo code as a cross section generator of BWR core calculations," Ann. Nucl. Eng., 32, 857 (2005).

[53] J. E. Hoogenboom, V. A. Khotylev, and J. M. Tholammakkil, "Generation of Multi-Group Cross Sections and Scattering Matrices with the Monte Carlo Code MCNP5," M\&C/SNA 2007-Mathematics \& Computations and Supercomputing in Nuclear Applications, Monterey, CA (2007).

[54] R. J. J. Stamm'ler and M. J. Abbate, "Methods of SteadyState Reactor Physics in Nuclear Design," Academic Press, London (1983).

[55] H. J. Park et al., "Qualification of McCARD/MASTER System for YGN 4," Trans. KNS, Taebak-Si, Korea, May 26-27 (2011).

[56] B. O. Cho et al., "MASTER-2.0: Multi-purpose Anlayzer for Static and Transient Effect of Reactor," KAERI/TR1211/99, Korea Atomic Energy Research Institute (1997).

[57] M. Tohjoh, et al., "Effect of Error Propagation of Nuclide Number Densities of Monte Carlo Burnup Calculations," Ann. Nucl. Eng., 33, 1424 (2006).

[58] N. Garcia-Herranz, "Propagation of statistical and nuclear data uncertainties in Monte Carlo burnup calculations," Ann. Nucl. Eng., 35, 714 (2008)

[59] H. J. Shim, et al., "Monte Carlo Adjoint Sensitivity and Uncertainty Analysis for Nuclear Data Covariance," ICNC 2011-International Conference on Nuclear Criticality, Edinburgh, Scotland, Sep. 19-22 (2011).

[60] I. Kodeli, "SUSD3D: A Multi-Dimensional, DiscreteOrdinates Based Cross Section Sensitivity and. Uncertainty Analysis Code System," RSICC Code Package: CCC-695 
(2000).

[61 ] H. Rief, "Generalized Monte Carlo Perturbation Algorithms for Correlated Sampling and a Second-Order Taylor Series Approach," Ann. Nucl. Energy, 11, 455 (1984).
[62] Y. Nagaya, T. Mori, "Impact of Perturbed Fission Source on the Effective MultiplicationFactor in Monte Carlo Perturbation Calculations," J. Nucl. Sci. Technol., 42[5], 428 (2005) 